\title{
Investigation of luminescence excited by electrons from cold emission from a carbon nanotube cathode
}

\author{
Mateusz Mroczkowski, ${ }^{* 2}$ Jerzy Kalenik, $^{2}$ Elżbieta Czerwosz, ${ }^{1}$ Jan Szmidt ${ }^{2}$ \\ ${ }^{I}$ Tele\&Radio Research Institute, Department for Nanotechnology, ul. Dluga 44/50, 00-241 Warszawa, \\ ${ }^{2}$ Institute of Microelectronics and Optoelectronics, Warsaw University of Technology, Koszykowa 75, \\ 00-662 Warszawa
}

Received June 15, 2010; accepted June 28, 2010; published June 30, 2010

\begin{abstract}
Modern light sources are expected to have low power consumption, high efficiency of light emission and to be ecological. This demand creates a need to develop new materials and technologies to fabricate light sources that will replace traditional, high power consuming light sources. Such new sources may use the phenomenon of luminescence excited by electrons, from cold emission.

An investigation of cold electron emission from a carbon nanotube cathode was undertaken. As a part of this research, a nanostructural material built of carbon nanotubes was fabricated. A special test stand, including a four-section high voltage power supply, was assembled. The results will be used to anticipate the possibility of fabricating efficient light emitting devices based on such cathodes.
\end{abstract}

Growing worldwide energy consumption and shrinking deposits of fossil fuels created a need to replace devices and tools we use in everyday life with their low energy consuming equivalents. One type of such devices are light sources. It is estimated, that $20 \%$ to $50 \%$ of electrical energy consumption in homes and offices is due to lighting [1]. With overall luminous efficiency of a typical filament incandescent light bulb of $2 \%$ to $2.5 \%$ there is a lot of place for improvement and development of new materials and technologies to fabricate efficient light sources.

It is proposed that the phenomenon of cold emission can be utilized as a source of electrons in luminescent, low power consuming light sources. In our opinion a new type of composite carbonaceous nanomaterial cold cathode could be applied in such a device.

\section{CARBON NANOTUBES (CNT) FILM AS A COLD EMISSION ELECTRON SOURCE (CES)}

Cold emission electron sources are devices that can emit electrons at ambient temperature. Such sources use only applied electric field overcome work function of the source's material. So far there have been a few attempts to create carbon-based electron sources utilizing field effect for electron emission [3]-[6]. One of such example are Spindt-type sources, researched by NEC company in Japan and SRI company in USA. Spindt-type CES with

*E-mail: m.mroczkowski@x.wp.pl
Diamond-like Carbon films seem to be unstable and not efficient enough. Additionally they require expensive electrono-litography as a part of the fabrication process.

To obtain an electron source that can be driven by relatively low voltage it should be fabricated from a material with low work function. Another method is to shape the surface of the cathode in a way that will locally increase charge density. Carbon nanotubes can have the length-to-diameter ratio of up to $132 \times 106$ [2], which suggests that they can be utilized to increase charge density. Additionally, the work function of films containg CNT is about $50 \%$ lower than that of diamond-like carbon or other carbonaceous films. Carbon nanotube CES have been so far utilized by Samsung company as a cathode in one TV set model.

CNT cathodes that were under investigation were fabricated with well known technologies like Physical Vapour Deposition (PVD) or Chemical Vapour Deposition (CVD). The utilization of such techniques allows to lower the production costs of cathodes. The fabrication was a two step process.

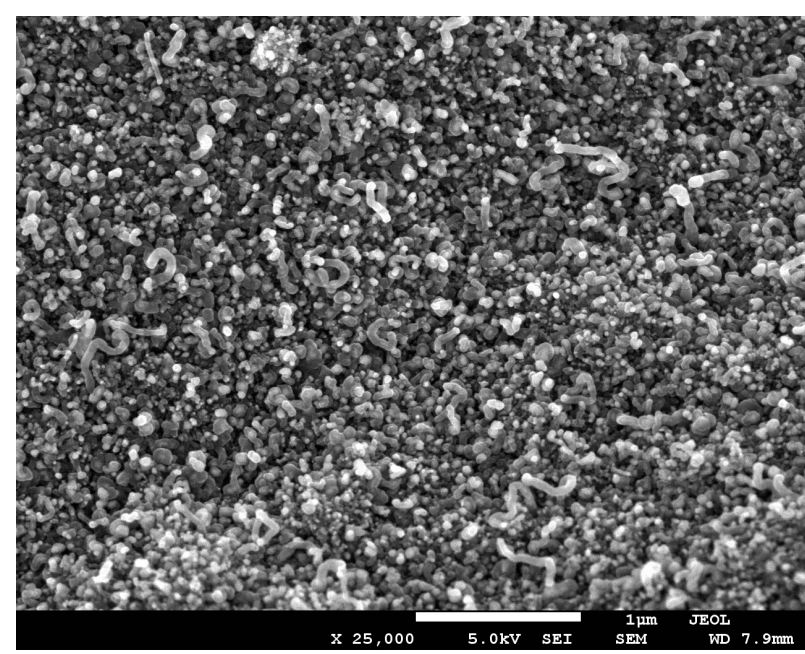

Fig. 1. Scanning electron microscope (SEM) image (25·103 times magnification) of obtained CNT films showing the distribution of CNT over the substrate. 
In the first step, using PVD a film of carbonaceous nanomaterial and nickel were deposited on a silicon substrate. In the second step the structure was annealed at high temperature. During the annealing, in the atmosphere of argon and xenon carbon nanotubes were formed on the substrate. Obtained NCTs were $35 \mathrm{~nm}$ to $45 \mathrm{~nm}$ in diameter. The amount of CNT over a unit of cathode's surface cannot be too high, otherwise it would worsen the emission capabilities of the cathode. If CNT are too densely distributed over the surface it uniforms the electric filed and therefore lowers the amount of places with increased charge density.

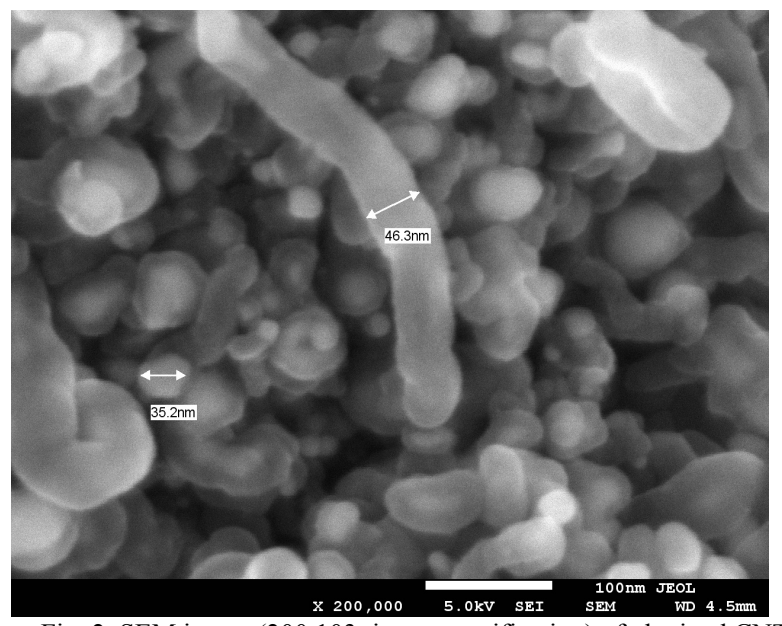

Fig. 2. SEM image (200·103 times magnification) of obtained CNT films showing fabricated nanotubes.

\section{TEST STAND FOR INVESTIGATION OF COLD EMISSION}

To study the cold emission of electrons from carbonaceous nanomaterial cathodes a special test stand was assembled. It consists of two vacuum chambers and a special four-section high-voltage regulated power supply.

The first vacuum chamber was used for measurements of emission current. It consisted of a stand for the cathode and a mechanism, that allowed to regulate the distance from the cathode and the anode.

The second chamber was used to observe the phenomenon of luminescence excited by electrons from carbon nanotube CES. In this chamber a CNT cathode was placed in a special stand behind a round luminescent screen. This stand allowed to place the cathode in a desired distance from the screen and ensure that both the cathode and the screen are parallel.

To obtain voltages required for cold emission a special power supply was assembled. It consisted of 4 independently regulated constant current power supplies. There were three high voltage sections, with output voltages of $20 \mathrm{kV}, 12 \mathrm{kV}$ and $5 \mathrm{kV}$, and one low voltage section with a negative voltage output of $-200 \mathrm{~V}$. The maximal output current for each section was $5 \mathrm{~mA}$. In the initial measurements only one high voltage section was utilized to power the test stand. The configuration of the power supply allows it to be used to power a prototype light source with carbon nanotube cathode with electrode configuration similar to a classic cathode ray tube.

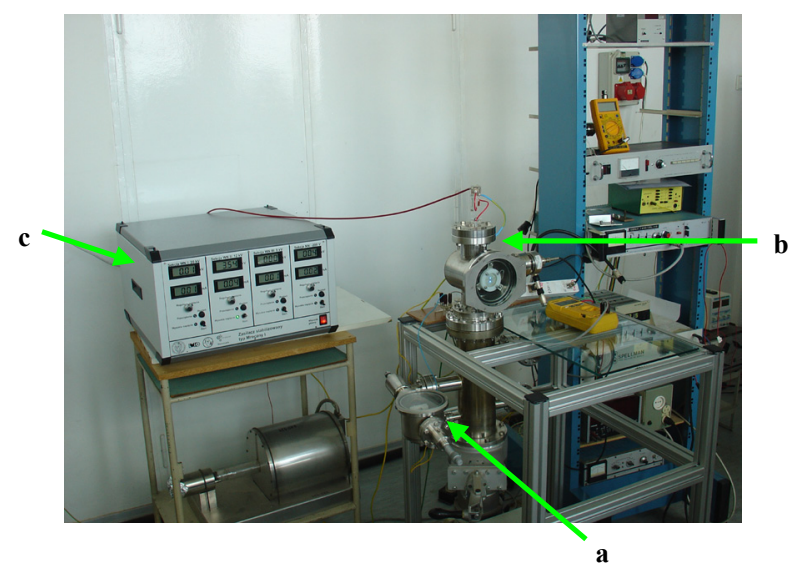

Fig. 3. A photograph of the test stand: a) the chamber for emission current measurement, b) the chamber for observation of luminescence, c) high-voltage power supply.

\section{RESULTS}

With carbon nanotube cathodes fabricated as described in Paragraph 1, there were some measurements performed. Emission current from the cathode and luminous power were estimated.

\subsection{Emission current}

The measurements of emission current show that there are two threshold voltages for the cold electron emission. Below the lower threshold voltage there is no emission. For voltages from between both threshold voltages emission current was practically negligible. For voltages above the higher threshold value the emission current changed proportionally to applied voltage.

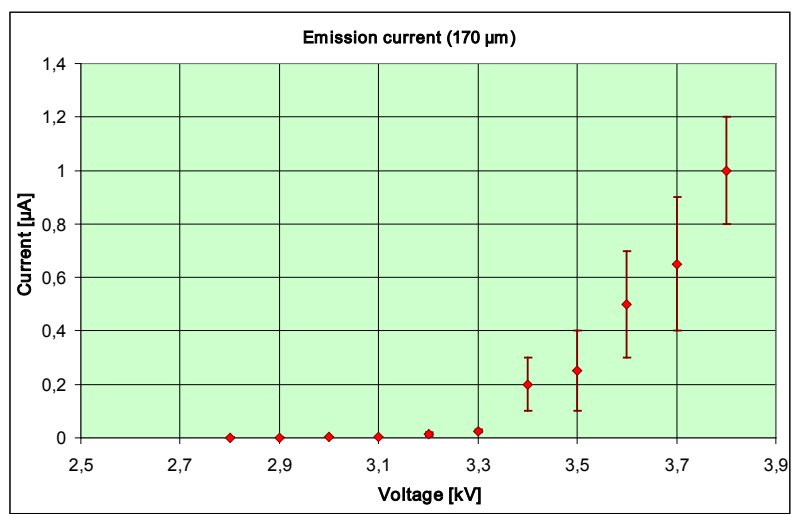

Fig. 4. Emission current as a function of voltage. 
For a CNT cathode placed in a distance of $170 \mu \mathrm{m}$ from the test stand anode, the lower threshold voltage was about $2.8 \mathrm{kV}$. For voltages of $2.8 \mathrm{kV}$ to $3.3 \mathrm{kV}$ the emission current value was measured in single nA. For voltages above $3.3 \mathrm{kV}$ the emission current rose and reached a maximal value of $1.2 \mu \mathrm{A}$ for $3.8 \mathrm{kV}$.

\subsection{Luminous power}

The luminous power of light emission from a blue phosphor, exited by electrons emitted from the carbon nanotube cathode, was estimated. In Fig. 5 there is a relation between estimated luminous power and applied voltage. No light emission was observed for voltages below $2 \mathrm{kV}$. Above this voltage there was weak light emission observed (up to 0.1 a.u.). For voltages above $3 \mathrm{kV}$ estimated luminous power rose, showing a similar relation to applied voltage to that of emission current.

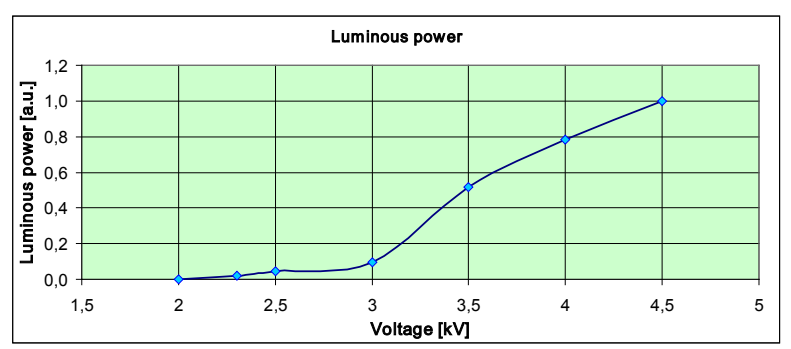

Fig. 5. Estimated luminous power as a function of voltage.
In conclusions, carbon nanotube cathodes for cold electron emission were fabricated using well known techniques, like PVC and CVD. Measurements of emission current as a function of applied voltage were performed. Luminous power from phosphor excited by electrons from fabricated cathode was estimated.

In both cases - emission current and luminous power two threshold voltages were observed. Above the first threshold voltage, the emission of electrons or light was initialized, but its value was low. Above the second threshold voltage, the emission of electrons or light started to rise rapidly as a function of voltage.

These initial measurements allow to anticipate that it's possible to fabricate efficient CES based on CNT films, that can be utilized in a new type of a light emitting devices.

This work was performed as a part of MNT/08/2009 Nanostructural carbonaceous films for cold emitters (NANOCAFE) research grant.

\section{References}

[1] P. Hawken, A. Lovins, L. H. Lovins, Natural Capitalism (Back Bay Press, Time Warner Book Group 2000).

[2] X. Wang, Q. Li, J. Xie, Z. Jin, J. Wang, Y. Li, K. Jiang, S. Fan, Nano Letters 9(9), 3137 (2009).

[3] Y. Saito, S. Uemura, Carbon 38, 169 (2000).

[4] Y. Cheng, O. Zhou, C. R. Physique 4, 1021 (2003).

[5] G. A. J. Amaratunga, S. R. P. Silva, Appl. Phys. Lett. 68, 2529 (1996).

[6] A. V. Karabutov, V. D. Frolov, S. M. Pimenow, V. I. Konov Diamond and Rel. Mat. 8, 763 (1999). 